\title{
Design and Improvement of Devices for Preparing Film Materials
}

\author{
KEGAO Liu ${ }^{\mathrm{a}^{*}}, \mathrm{CHAO} \mathrm{Xu}^{\mathrm{b}}$, YONG $\mathrm{Xu}^{\mathrm{c}}$ and LEI shi ${ }^{\mathrm{d}}$ \\ School of Materials Science and Engineering, Shandong Jianzhu University, Fengming Road, Jinan \\ 250101, China \\ aliukg163@163.com, ${ }^{\mathrm{b}} 450162281 @ q q . c o m,{ }^{c}$ xuyong2612@gmail.com, ${ }^{\mathrm{d}}$ slcqj@sdjzu.edu.cn
}

\begin{abstract}
Most equipments for film material preparation are more expensive, so it is necessary to design some low cost, practical instrument or devices. This work gives three kinds of devices for preparing film matreials. One is the experimental apparatus for reducing and vulcanization, the others are a vulcanization and exhaust gas treatment apparatus, and an experimental apparatus for sulfur deposition film. The details for their principles and structures are reported.
\end{abstract}

Keywords: Design, improvement, devices, film material

\section{Introduction}

In recent years, the study on functional thin film materials has become a hot topic [1-4], especially in the aspect of thin film photovoltaic materials [5-6]. The related preparation equipments and methods are very expensive [7-8], it is the obstacle to develop these functional film materials. Therefore, some instruments or devices need to be design for lower cost and practical use. Three kinds of devices for preparing film materials are designed in this work.

\section{An Experimental Device for the Film Reduction and Oxidation}

In the present technology, a known technique is that reduction and vulcanization processes are carried out step by step in laboratory, so that the experimental period is longer with complex experiment operation, and the experimental temperature is not easy to be regulated and controlled, it makes the workload greater. Toxic gases dischared directly in experimental process esily cause serious air pollution in the laboratory.

The device relates to an experimental apparatus for reduction and vulcanization, and particularly to an experimental apparatus that not only exhaust gas and unreacted reducing harmful gases can be collected during the reaction and vulcanization, but also the appartus has the function of automatic temperature control.

* Corresponding author: liukg163@163.com 
The purpose of designing this device is to overcome the shortcomings of present technologies, it can provide an experimental closed apparatus for chemical reduction and vulcanization, the temperature can be automatically controlled and the exhaust gas and unreacted reducing harmful gases can be collected during the experiments. This experimental device has a strong practical and promotional value due to its many advantages of simple structure, easy operation and low cost.

The technical project of this device to solve the technical problems is adopted as following: The main components include heating plate, flasks, quartz tube, vacuum pump, air bags, temperature controller, tubular resistance furnace. The flasks are placed on top of the heating plate, an appropriate amount of reducing agent hydrazine hydrate in erlenmeyer flask can be heated by heating pan; The flasks and tubular resistance furnace are connected by the glass tube and a hose, the hydrazine vapor can go into the quartz tube through the glass tube and hoses erlenmeyer flask, and then forms a reducing atmosphere. When the tube resistance furnace is power on its temperature will rise, which makes the sulfur powder in quartz tube sublimate to form sulfur atmosphere. And temperature controller connected with the tube resistance furnace is to adjust the temperature for reduction and curing reaction, and the base below the tubular resistance furnace fixs and supports the whole device to ensure the stability. At the right of quartz tube the air bag is used for collecting the reaction of hydrazine discharged steam, sulfur vapor and unreacted gas; The vacuum apparatus at the right of the entire apparatus can evacuate before the reaction, it may reduce the oxygen content and the oxidation of samples.

The beneficial effects of the utility model are as follows: simplifying the experimental procedure and shortenning the test cycle, reducing test costs. In addition simple equipment increases the controllability of the experimental conditions, but it also reduces contamination of the laboratory environment.

Fig.1 shows the front view of the utility model structure diagram of a specific embodiment. In above two figures it inconludes the following parts, 1 is a sample, 2 a sulfur powder, 3 the flask, 4 the heating plate, 5 a temperature controller, 6 a base, 7 a vacuum pump, 8 the balloon, 9 a quartz tube, 10 a tube resistance furnace.

In order to clearly illustrate the technical characteristics of this program, the program will be explained through the following specific embodiment with additional figures. In Fig.1, the base beneath tubular resistance furnace supports the entire reactor. Vacuum pump evacuates for the entire apparatus. Heating disc is placed below the Erlenmeyer flask heats reducing agent in the bottle. The temperature controller is connected with tubular resistance furnace controls the entire reduction and vulcanization reaction temperature. A quartz tube in tube furnace provides a sealed environment for reduction and curing reaction. Toxic gas and unreacted gas generated during the reaction are collected by airbag at the right end of the quartz tube. 


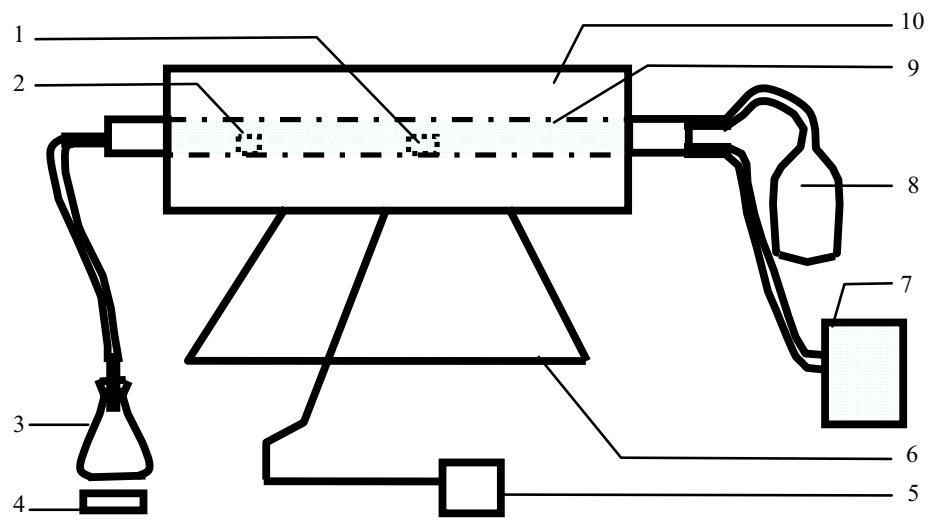

Fig.1 Schematic structure of the experimental apparatus for reduction and oxidation

\section{An Curing and Exhaust Gas Treating Experimental Apparatus}

The utility model relates to an exhaust gas treatment and curing experimental device, and more particularly to a field of absorbing, process emissions and harmful gases unreacted sulfide produced during the reaction, but also to good insulation function of the experimental apparatus for its production, research and development.

In the prior art, it is known that the vulcanization process technology lab, require higher temperatures and long reaction time, so it has higher requirements for laboratory equipments and a long test cycle, reduces the efficiency of the vulcanization reaction. Experimental processes produce toxic gases by direct emissions, it is likely to cause air pollution nearby laboratory.

The purpose of this utility model is to provide a curing reaction experimental device for the deficiencies of the prior art, it does not require a high temperature environment, and can absorb and process the harmful or unreacted gas generated during experiments. This experimental device is simple, easy operation, low test cost, has strong practicability and promotional value.

The project to solve the technical problems of the utility model is adopted as follows: The main components include heating plate, flasks, autoclave, insulation sleeve, porous sample holder, porous sample holder, exhaust gas treatment apparatus. Flasks hydrazine hydrate, reaction vessel and sulfur powder are heated by the heating plate respectively, Erlenmeyer flasks and autoclave are connected by the glass and the hoses; The sample holder and porous micro sample holder are placed in the reactor; The reactor outer is covered with insulation sleeve; The reactor is connected via a conduit with the exhaust gas treatment device. After the hydrazine hydrate steam enters into the reactor through the glass and hoses, it will form vulcanization atmosphere by the reaction of sulfur powder with hydrogen from the thermal decomposition of hydrazine hydrate; The reactor with outer insulation sleeve can prevent heat loss and keep the reactor at high temperature for vulcanization reaction required; The sulfur powder and sample porous holder are placed in the reactor from the bottom to top, the vapor can pass through the sample holder to make the sample be vulcanized; The reactor connected exhaust gas treatment device via a catheter at right. The exhaust gas treatment apparatus is used for absorbing and collecting the gas discharged in reaction, the treated gas is discharged from the right end of the exhaust port.

The utility model has beneficial effects: no high temperature conditions, simple experimental equipment, and low cost in the experiment. In addtion, it has simple 
experimental procedure and is easy to operate, it increases the operability of experimental conditions and well protectes the experimental environment.

Fig.2 is a front view of this specific embodiment of the utility model structure.

In Fig.2, part 1 is the heating plate, 2 a conical flask, 3 the microporous sample holder, 4 sulfur powder, 5 insulation sets, 6 porous sample holder, 7 the sample, 8 the reactor, 9 an exhaust gas treating solution, 10 the exhaust port.

For clearly to illustrate the technical characteristics of this program, later it will be explained through a specific embodiment.

In Fig.2, the heating plates were placed below the erlenmeyer flask and reactor, they heat the reducing agent and sulfur powder bottle inside the reactor, and provide heat for the sulfurization reaction. The insulation sleeve of the reactor outer is used to prevent the kettle heat disperses quickly to ensure that the vulcanization reaction at a certain temperature. Microporous sample holder inside the reactor and the porous sample holder are placed with sulfur powder and the sample to be vulcanized, it can make the vulcanization atmosphere pass, to ensure complete vulcanization reaction. Gas and unreacted harmful gases generated during the reaction from the conduit enter into the exhaust gas treating solution for processing and then discharged from the exhaust port.

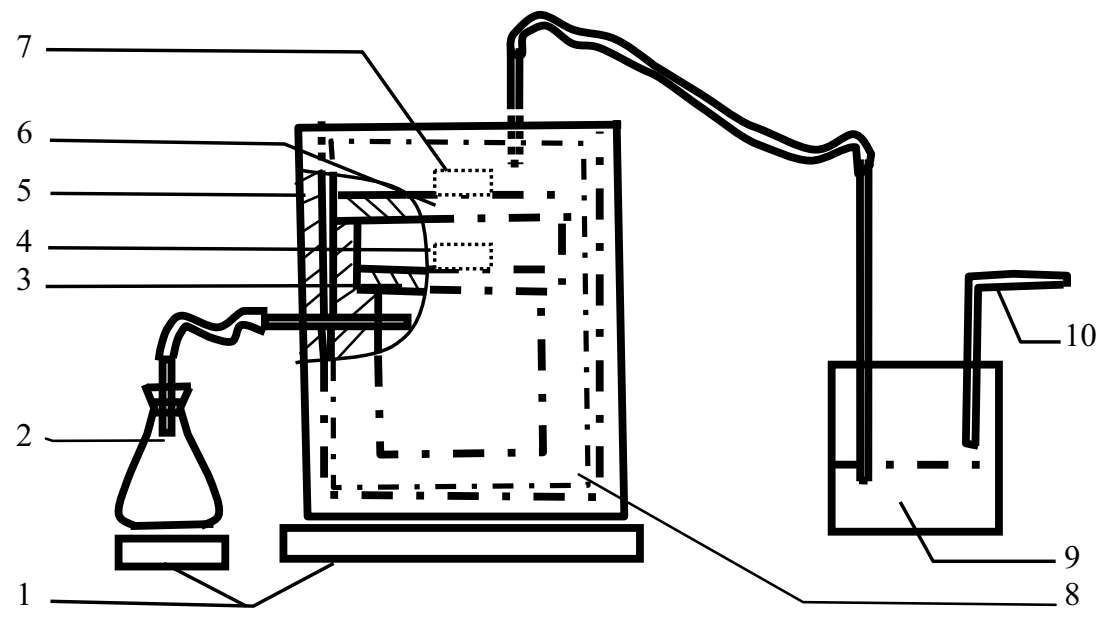

Fig.2 The schematic structure of sulfide and gas treatment experimental equipment

\section{An Experimental Apparatus for Sulfur Deposition}

The utility model relates to a method of depositing sulfur experimental apparatus, particularly to a field of experimental apparatus for absorbing, processing unreacted harmful exhaust gases produced during the reaction.

In the prior art technology, it is known that sulfur film deposition in laboratory process requires high temperatures and long reaction time, so it leads to higher requirements for laboratory equipment and a long test cycle, reduces the deposition efficiency. Experimental process produces toxic gases with direct emissions, is likely to cause air pollution nearby laboratory.

For the deficiencies of the prior art the utility model's purpose is to provide sulfur film deposition experimental device which does not require high temperature environment, and can absorb the harmful gas and unreacted gas generated during processing experiments. 
Experimental device is simple, easy operation, low cost test, and has a strong practical and promotional value.

The technical solution to solve the technical problems of the utility model is adopted: The main components include heating coil, reactor, thermoelectric cooler, porous sample holder, exhaust gas treatment devices. The reactor was placed over the heating plate, hydrazine hydrate as reducing agent and elemental sulfur are heated by the heating plate in the autoclave; The porous sample holder with sulfur powder is placed within the reactor, which can make hydrazine vapor pass through to form mixed steam atmosphere; The reactor, thermoelectric coolers and hoses are connected by a glass, through the glass hoses and the erlenmeyer flask, mixed vapor enters into thermoelectric cooler; The sample substrate was placed within the thermoelectric cooler, after power on, the cooler lower the temperature, the sulfur vapor will be deposited on the substrate to forming a sulfur film; On the right side the thermoelectric cooler is connected with an exhaust gas treatment apparatus for absorbing and collecting processing the reaction gas released, the treated gas is discharged from the right end of the exhaust port.

The utility model has the beneficial effects: no high temperature conditions, simple test equipment and low experimental cost. Further, the experimental procedures are simple and easy to operate, it increases the operability of the experimental conditions and the experimental environment will be well protected.

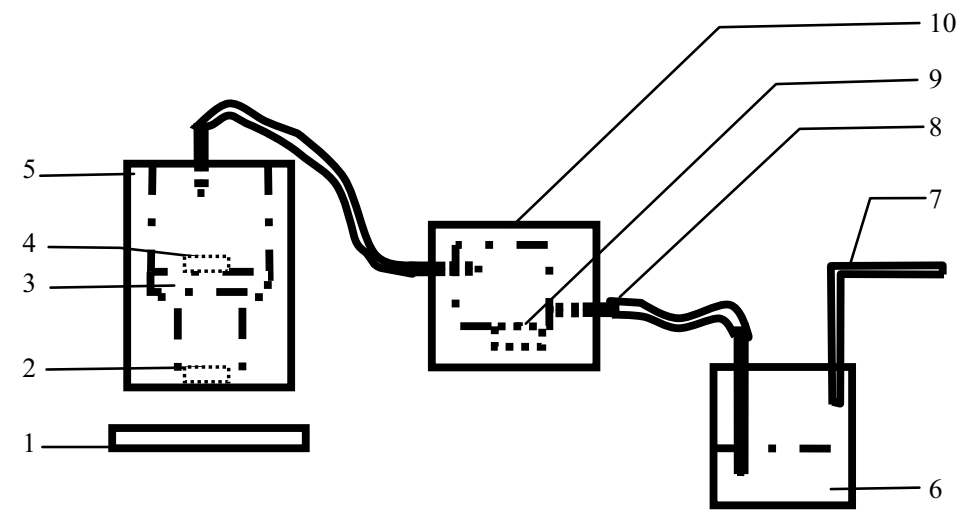

Fig.3 The schematic structure of the experimental apparatus for depositing sulfur film

Fig. 3 is the front view for a specific embodiment of the utility model structure diagram.

In Fig. 3, the part 1 is the heating plate, 2 a hydrazine hydrate, 3 a porous sample holder, 4 sulfur powder, 5 the reactor, 6 an exhaust gas treatment liquid, 7 the exhaust port, 8 an exhaust gas discharge port, 9 the sample substrate, 10 a thermoelectric cooler.

For clearly to illustrate the technical characteristics of this program, it will be explained through the following specific embodiment. In Fig.3, a heating plate is disposed below the reaction vessel, hydrazine hydrate and sulfur powder are heated in the autoclave. The porous sample holder with sulfur powder is placed inside the reactor, it permits a reducing atmosphere pass through to form mixed vapor atmosphere within the reactor. Through the glass hoses mixed vapor enters into the thermoelectric coolers, with decreasing temperature of the thermoelectric cooler, the sample substrate can be deposited to form the sulfur film. from the discharge port, Gas and unreacted harmful gases generated during the reaction discharged, then through the catheter into the exhaust gas treating liquid for processing, and then discharged from the exhaust port. 


\section{Summary}

To reduce the production cost of the film materials, it designs three kinds of practical devices for preparing films: A reduction and the experimental device for vulcanization, a vulcanization and flue gas treatment test apparatus and a film deposition of sulfur experimental device. It in detail to introduce their principles, structures and operation methods.

\section{Acknowledgment}

This work was financially supported by the National Natural Science Foundation of China (No.51272140).

\section{References}

1. X.X. Liu, H.J. Chen, S.T. Tan, Overview of high-efficiency organic photovoltaic materials and devices, Renewable and sustainable energy reviews, 52 (2015) 1527-1538.

2. P. Rai, S. Oh, M. Ramasamy, V.K. Varadan, Photonic nanometer scale metamaterials and nanoporous thermoelectric materials for enhancement of hybrid photovoltaic thermo- electric devices, Microelectronic Engineering, 148 (2015), 104-109.

3. D.M. Dong, W.W. Wang, G.B. Dong, et al, Electrochromic properties of $\mathrm{NiO}_{\mathrm{x}}$ : $\mathrm{H}$ films deposited by $\mathrm{DC}$ magnetron sputtering for $\mathrm{ITO} / \mathrm{NiO}_{\mathrm{x}}$ : $\mathrm{H} / \mathrm{ZrO}_{2} / \mathrm{WO}_{3} / \mathrm{ITO}$ device, Applied Surface Science, 357 (2015) 799-805.

4. A. Barranco, A. Borras, A.R. Gonzalez-Elipe, A. Palmero, Perspectives on oblique angle deposition of thin films: From fundamentals to devices, Progress in Materials Science, 76 (2016) 59-153.

5. D.Y. Park, D.H. Nam, S.H. Jung et al. Optical characterization of $\mathrm{Cu}_{2} \mathrm{ZnSnSe}_{4}$ grown by thermal co-evaporation. Thin Solid Films, 519 (2011) 7386-7389.

6. S. Xia, Synthesis of $\mathrm{CuInS}_{2}$ and $\mathrm{Cu}_{2} \mathrm{ZnSnSe}_{4}$ nanostructures in polyol and its film preparation by ink, Doctor thesis of Hangzhou, Zhejiang University, 2010.

7. C. Lopes, M. Vieira, J. Borges, et al, Multifunctional Ti-Me ( $\mathrm{Me}=\mathrm{Al}, \mathrm{Cu})$ thin film systems for biomedical sensingdevices. Vacuum, 122 (2015) 353-359.

8. C. Prahoveanu, A. Lacoste, S. Béchu, et al, Investigation of $\mathrm{Mg}_{2}(\mathrm{Si}, \mathrm{Sn})$ thin films for integrated thermoelectric devices. Journal of Alloys and Compounds, 649(2015) 573-578. 